\title{
Transport spectroscopy of induced superconductivity in the three-dimensional topological insulator HgTe
}

\author{
Jonas Wiedenmann, ${ }^{1, *}$ Eva Liebhaber, ${ }^{1}$ Johannes Kübert, ${ }^{1}$ Erwann Bocquillon,${ }^{1,2}$ Pablo Burset, ${ }^{3}$ Christopher Ames, ${ }^{1}$ \\ Hartmut Buhmann, ${ }^{1}$ Teun M. Klapwijk, ${ }^{4}$ and Laurens W. Molenkamp ${ }^{1}$ \\ ${ }^{1}$ Experimentelle Physik III, Physikalisches Institut, Universität Würzburg, Am Hubland, D-97074 Würzburg, Germany \\ ${ }^{2}$ Laboratoire Pierre Aigrain, Département de Physique de l'ENS, École Normale Supérieure, PSL Research University, \\ Université Paris Diderot, Sorbonne Paris Cité, Sorbonne Universités, UPMC Université Paris 6, CNRS, 75005 Paris, France \\ ${ }^{3}$ Department of Applied Physics, Aalto University, 00076 Aalto, Finland \\ ${ }^{4}$ Kavli Institute of Nanoscience, Faculty of Applied Sciences, Delft University of Technology, Lorentzweg 1, 2628 CJ Delft, Netherlands
} (Received 3 June 2017; published 10 October 2017)

\begin{abstract}
The proximity-induced superconducting state in the three-dimensional topological insulator HgTe has been studied using electronic transport of a normal metal-superconducting point contact as a spectroscopic tool (Andreev point-contact spectroscopy). By analyzing the conductance as a function of voltage for various temperatures, magnetic fields, and gate voltages, we find evidence, in equilibrium, for an induced order parameter in $\mathrm{HgTe}$ of $70 \mu \mathrm{eV}$ and a niobium order parameter of $1.1 \mathrm{meV}$. To understand the full conductance curve as a function of applied voltage we suggest a non-equilibrium-driven transformation of the quantum transport process where the relevant scattering region and equilibrium reservoirs change with voltage. This change implies that the spectroscopy probes the superconducting correlations at different positions in the sample, depending on the bias voltage.
\end{abstract}

DOI: 10.1103/PhysRevB.96.165302

\section{INTRODUCTION}

The two most important methods to obtain reliable quantitative spectral information about the electronic properties of a superconductor are Giaever tunneling [1] and point-contact Andreev spectroscopy [2,3]. In tunnel spectroscopy two thin metal films are weakly coupled by an insulating tunnel barrier, leading to a current-voltage characteristic which is controlled by the unperturbed superconducting densities of states in both metals $N_{s}(E)$ and their occupation, given by the Fermi functions $f_{0}(E)$. The technique can also be used successfully to study the proximity effect in superconducting bilayers, as experimentally shown by Wolf and Arnold [4], but requires the difficult development of an opaque tunnel barrier. The second method, point-contact Andreev spectroscopy, has become a standard tool to evaluate the microscopic properties of new bulk materials. The experimental configuration consists of a macroscopically sized point-shaped metal wire, which touches a superconducting material, usually a single crystal. In the contact area the conductance in both the superconducting and normal regimes is dominated by the channels with the highest transmission, usually loosely called pinholes. Thus, there is no need to know the exact nature of the contacting layer, and the transmissivity of the point contact can be assumed to reach values on the order of 1 without disturbing the properties of the superconductor. This latter assumption is valid because the two bulk materials are connected by an area which is very small compared to the lateral dimensions of the materials and assumed to be smaller than the elastic mean free path of both materials (ballistic transport). Such a geometry leaves the reservoirs undisturbed, a crucial condition for the determination of the electronic parameters of the

\footnotetext{
*jonas.wiedenmann@physik.uni-wuerzburg.de
}

superconductor and generalized in the Landauer-Büttiker picture of quantum transport.

Our aim in this paper is to apply Andreev spectroscopy to the proximity-induced superconducting state in a $3 \mathrm{D}$ topological insulator (3DTI). The application of Andreev spectroscopy to low-dimensional heterostructures is a much less mature experimental technique than for bulk systems. The point contact has to be lithographically defined and is therefore usually larger than for bulk systems, where accidentally formed pinholes of smaller dimensions dominate the transport. These experimental concerns are exacerbated in the case of spectroscopy on proximity-induced superconductivity because of the need to use two dissimilar materials and, unavoidably, a complex lithographically structured geometry. In fact, very few successful spectroscopic experiments on proximitized systems have been carried out. One example, on diffusive systems, is by Scheer et al. [5], using mechanical break junctions, an approach that merges bulk point-contact behavior with thin films. Recently, Kjaergaard et al. [6] presented results on point-contact spectroscopy in the ballistic Al/InAs system, which partially fulfills the experimental requirements. It shows the expected doubling of the quantized conductance steps for point contacts in the highly transmissive regime but exhibits, from a spectroscopic perspective, many puzzling results and, additionally, unexpected behavior as a function of the tunable point-contact transmissivity. A different geometry was used by Zhang et al. [7], also employing a tunable point contact, predominantly in the regime of low transmission.

We report on a study of a high-quality three-dimensional topological insulator, epitaxially grown strained $\mathrm{HgTe}$, which is proximitized by a conventional superconductor, niobium. In previous experiments we reported on the observation of a "missing $n=1$ " Shapiro step [8], an indication of an unconventional Josephson effect in 3DTI HgTe-based Josephson junctions. The same type of observation was subsequently done in Josephson junctions in a two-dimensional topological 
insulator showing a sequence of even-only Shapiro steps (up to $n=10$ ) and emission at half the Josephson frequency. Both signatures indicate at least a fractional $4 \pi$-periodic Josephson effect and point towards the presence of gapless MajoranaAndreev bound states [9,10]. Since the Josephson effect arises from the proximity-induced superconducting state, we are interested in a determination of the energy-dependent properties of this induced superconducting state, which, in principle, serves as a coherent reservoir for the Josephson effect, analogous to the established proximity-effect-based niobium superconductor-insulator-superconductor (SIS) junctions [11]. It is crucial to be able to measure these electronic states directly, particularly because the Josephson effect itself contains information only about the phase difference and the nature of the current-phase relation and not about its energy dependence. For this reason we designed an experiment which is based on an $\mathrm{NcS}_{\mathrm{p}}$ point contact to emulate Andreev spectroscopy of the induced superconducting state ( $\mathrm{N}$ is a normal reservoir, which in our case is a topological insulator, $\mathrm{c}$ is the constriction, and $\mathrm{S}_{\mathrm{p}}$ is the proximity-induced superconductor), as schematically shown in Fig. 1(a). Therefore, the strained HgTe is defined lithographically to a finite-sized bar and covered over a small distance by a conventional superconductor $\mathrm{S}_{\mathrm{m}}$. We assume that an induced superconducting state exists underneath the superconducting material, which we label $S_{p}$. The electronic states in this region are the source for the observed Josephson effect. Note that in such a geometry no Majorana zero modes are expected to emerge due to the lack of confinement [12], but unconventional superconducting correlations might be observable $[13,14]$. We find that the electronic transport between the $\mathrm{N}$ reservoir and the $\mathrm{S}_{\mathrm{m}}$ reservoir is governed by two energy scales, which we identify as the superconducting gap of the niobium film $\Delta_{\mathrm{Nb}}$ and the induced gap in the surface states of the HgTe, labeled $\Delta_{\mathrm{p}}$. By using modeling as introduced by Blonder et al. [2] we are able to show that the transmissivity at the $\mathrm{HgTe} / \mathrm{Nb}$ interface is rather low. We argue that the voltage-carrying state, needed to obtain spectroscopic information, leads to a nonequilibrium occupation of the proximity-induced superconducting state, sending the device into different experimental conditions, depending on the bias voltage.

\section{SAMPLE DESCRIPTION}

The NcS junctions in this work are based on epitaxially grown layers of strained $\mathrm{HgTe}$ sandwiched between $\mathrm{Hg}_{0.3} \mathrm{Cd}_{0.7} \mathrm{Te}$ capping layers. These additional layers have a conventional band structure and protect against surface oxidation, which reduces the carrier mobility. They also protect the strained $\mathrm{HgTe}$ during subsequent lithographic processing. The HgTe sandwich is shaped as a 1- $\mu \mathrm{m}$-wide bar which at one or both ends tapers out at an angle of about $45^{\circ}$. The top $\mathrm{Hg}_{0.3} \mathrm{Cd}_{0.7} \mathrm{Te}$ capping layer is removed by dry etching and subsequently covered by niobium, which is in contact with the strained HgTe. Figure 1(a) shows a schematic drawing of the device. The orange part is the source superconductor $\mathrm{S}_{\mathrm{m}}$, made of niobium, and the blue part is the strained $\mathrm{HgTe}$. At the interface we allow for a finite-transmission coefficient, which is labeled $Z_{\mathrm{m}}$. This dimensionless barrier is, in general, connected to the normal-state transmission by $t=\left(1+Z^{2}\right)^{-1}$.
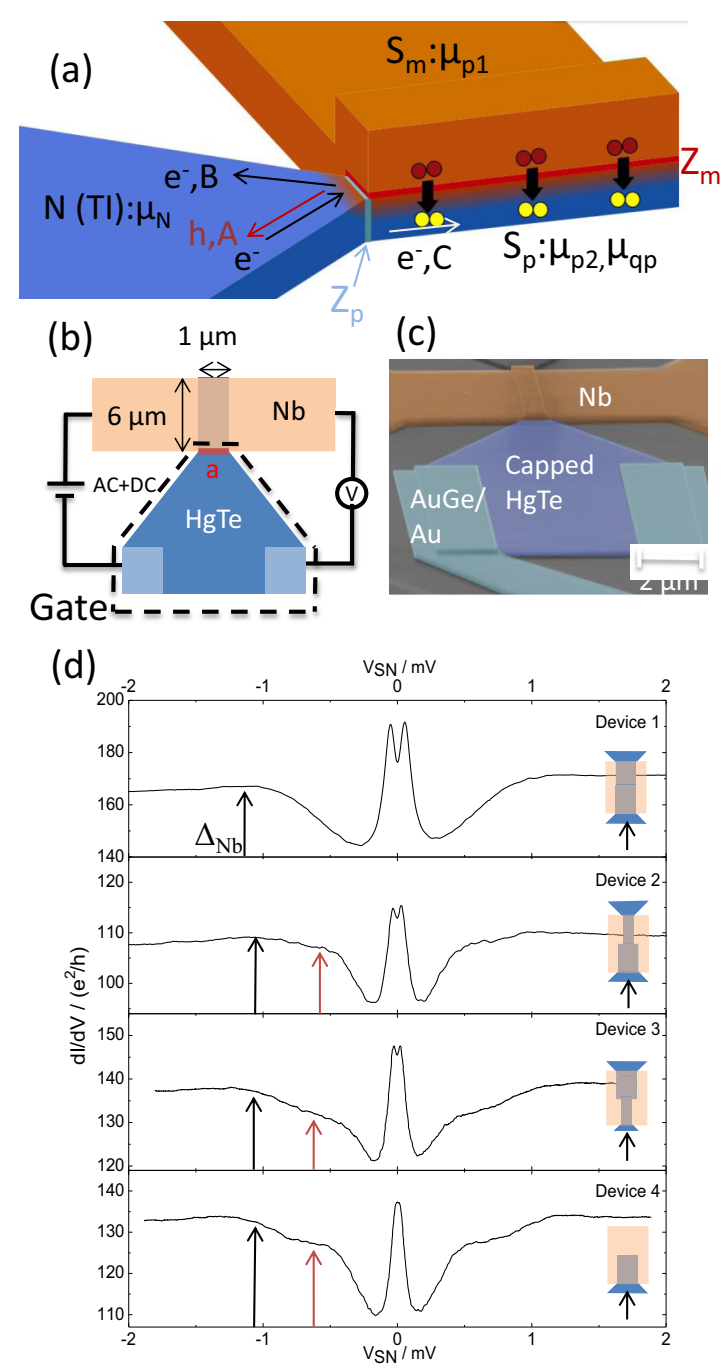

FIG. 1. (a) Schematic of the experiment: An $s$-wave superconductor $\mathrm{S}_{\mathrm{m}}$ (orange) is inducing superconducting pairing in an underlying topological insulator $S_{\mathrm{p}}$. This state is probed via a point contact. An electron impinging from the 3DTI reservoir $\mathrm{N}$ can either be Andreev reflected, normal reflected, or transmitted with probability amplitudes $\mathrm{A}, \mathrm{B}$, and $\mathrm{C}$ respectively. The current is carried away to the right of $Z_{\mathrm{p}}$ as a supercurrent. (b) Schematic of the device and measurement setup. A niobium strip is covering a $\mathrm{HgTe}$ bar which is coupled to an equilibrium reservoir via a small orifice marked with the letter $a$. The dashed lines mark the contours of the gate. (c) False-color SEM picture of a device without a gate electrode. (d) $d I / d V$ measurements of four devices. The devices differ by the "connectivity" of the $\mathrm{HgTe}$ bar, covered by niobium, as indicated in the inset.

The superconducting correlations are induced in the HgTe, indicated by yellow dots. The tapered part of the $\mathrm{HgTe}$, not covered by the niobium, is left capped by the $\mathrm{Hg}_{0.3} \mathrm{Cd}_{0.7} \mathrm{Te}$ layer, and we assume that this part has the same mobility as the starting material. At the constriction we allow for an additional elastic scattering parameter $Z_{\mathrm{p}}$.

The quality of the HgTe layers is characterized using a Hall bar fabricated from the same wafer. At zero gate voltage $\left(V_{g}=0\right)$ a density of $n_{2 \mathrm{D}} \approx 5 \times 10^{11} \mathrm{~cm}^{-2}$ and mobilities of $\mu \approx 200000 \mathrm{~cm}^{2} /$ Vs are routinely achieved, resulting in a mean free path $l_{\mathrm{mfp}} \approx 2-3 \mu \mathrm{m}$. The mobility is about 10 times 
lower when tuning the device into the $p$ regime. As shown in detailed magnetotransport studies $[15,16]$, clear quantum Hall plateaus are observed, indicating transport mediated predominately by two-dimensional states which were shown to originate from the topological surface states.

The point contact is fabricated using electron-beam lithography and poly(methyl methacrylate) resist. As HgTe is sensitive to temperatures above $90^{\circ} \mathrm{C}$, all bake-out and lift-off procedures are carried out well below this temperature. In the first step the HgTe mesa is defined using low-energy argon sputtering. During this process a thin titanium etch shield, separated by a $\mathrm{SiO}_{2}$ sacrificial layer from the HgTe, is protecting the mesa. The shield is afterwards removed by a buffered oxide etch dip. The dimensions of the mesa as shown in Figs. 1(b) and 1(c) are chosen such that the orifice ( $a=1 \mu \mathrm{m}$ for devices 1,2 , and 4 , and $a=0.6 \mu \mathrm{m}$ for device 3 , respectively) is smaller than the ballistic mean free path of the surface states. The size of the normal reservoir is much larger than this length scale to allow full energy relaxation in this region. In the next step, the superconductor is deposited. Since the interface is buried, the cap layer needs to be removed, which is done by argon etching, followed by in situ magnetron sputtering of about $110 \mathrm{~nm}$ of niobium. After this the leads for the Ohmic contacts are defined, and $50 \mathrm{~nm} \mathrm{AuGe/50} \mathrm{nm} \mathrm{Au}$ are deposited. The contact resistances are usually small $(<50$ $\Omega$ ). To allow control of the charge carrier density in the 3DTI a top-gate electrode is evaporated on top of the $\mathrm{HgTe}$ [see dashed lines in Fig. 1(b)] as follows. First, a thin $\mathrm{HfO}_{2}$ insulator is grown at about a temperature of $35^{\circ} \mathrm{C}$ via atomic layer deposition, followed by the deposition of $5 \mathrm{~nm} \mathrm{Ti} / 150 \mathrm{~nm}$ $\mathrm{Au}$. Using the same insulator on reference Hall bar structures, it is possible to tune the density from the $1 \times 10^{12} \mathrm{~cm}^{-2} n$-type regime to the $-1 \times 10^{12} \mathrm{~cm}^{-2} p$-type-dominated conductance. A false-color scanning electron microscopy (SEM) picture of a final device without an applied gate is shown in Fig. 1(c).

For the transport studies the samples are then cooled down in a dilution refrigerator with a base temperature of $30 \mathrm{mK}$ (device 1) or $120 \mathrm{mK}$ (devices 2-4), and the differential conductance $d I / d V$ is measured using low-excitation and low-frequency lock-in techniques combined with dc measurements, as depicted in Fig. 1(b). Several devices made from different wafers with and without a top gate have been measured, all yielding very similar results, from which four exemplary devices are discussed.

\section{METHOD OF ANALYSIS}

In the design of the experiment, we anticipate that the transport from $\mathrm{N}$ to $\mathrm{S}$ will be controlled by the process of Andreev reflection, which allows using the theory of Blonder et al. [2] (BTK theory). This theory assumes thermal equilibrium for the relevant states. In the experimental configuration used by us the occupation of states will potentially deviate from the equilibrium Fermi-Dirac distribution. As shown in Fig. 1(a), we define three sections through which the transport occurs in our device. In that drawing, the wide uncovered part of the 3DTI constitutes the $\mathrm{N}$ side and fulfills the criterion of a proper Landauer-Büttiker equilibrium reservoir with a Fermi function at the bath temperature $T_{b}$ and a Fermi level $\mu_{\mathrm{N}}=\mu_{\mathrm{p} 1}-e V_{\mathrm{SN}}$, which depends on the applied bias $V_{\mathrm{SN}}$. On the other side of the constriction, located at $Z_{\mathrm{p}}$, the main superconductor $\mathrm{S}_{\mathrm{m}}$, niobium, induces superconducting correlations in the 3DTI bar $S_{p}$. Both superconductors form the same macroscopic quantum state. The current through the sample, assumed to enter from the $\mathrm{N}$ part, is carried away as a supercurrent. Therefore, we do not expect a voltage drop beyond $Z_{\mathrm{p}}$, and the superconducting side is initially, for zero applied bias $V_{\mathrm{SN}}=0$, characterized by an equilibrium Fermi function $\mu_{\mathrm{p} 1}=\mu_{\mathrm{p} 2}$ at the bath temperature $T_{b}$. With this starting point we anticipate that the conductance as a function of voltage $V_{\mathrm{SN}}$ will, in principle, be described by

$$
\begin{aligned}
I_{S N}= & \frac{1}{e R_{N}} \int_{-\infty}^{+\infty}\left[f_{0}\left(E-e V_{\mathrm{SN}}, T\right)-f_{0}(E, T)\right] \\
& \times[1+A(E, Z)-B(E, Z)] d E,
\end{aligned}
$$

where $f_{0}(E, T)$ is the Fermi-Dirac distribution at energy $E$ and temperature $T . A(E)$ and $B(E)$ are the probability amplitudes for Andreev and normal reflection of an incident electron from and to the normal reservoir. The normal-state resistance $R_{N}$ is assumed to be the resistance arising from the finite number of modes carried by the cross section. The voltage drop is located at the orifice with elastic scattering parametrized by $Z=Z_{\mathrm{p}}$, as indicated in Fig. 1(a).

We do not know the coefficients $A(E)$ and $B(E)$ a priori. They contain the spectral information we are interested in and are the result of the interaction of the superconductor with the confined bar of the 3DTI with its limited geometry, finite elastic mean free path, and finite interfacial transparency $Z_{\mathrm{m}}$ $[5,17]$. In addition we need to consider that the normal part is a 3DTI, where helical surface states dominate the transport $[12,14,18]$.

In the covered TI bar we allow for a finite paring potential $\Delta$, which implies that the self-consistency equation of the Bogoliubov-de Gennes equations

$$
\Delta(\vec{r})=V_{N} \sum_{E>0} v^{*}(\vec{r}) u(\vec{r})\left[1-2 f_{0}(E)\right]
$$

needs to be fulfilled. The value $\Delta$ depends on the distribution function, which for a driven system may differ from the one assumed for equilibrium reservoirs.

Hence, we will analyze our data under the assumption that Andreev reflection, due to a finite value of $\Delta$, takes place at $Z_{\mathrm{p}}$, which allows us to apply Eq. (1) to our system with, initially, for low voltages, the equilibrium reservoirs taken to be on the normal side and in the proximitized HgTe on the superconducting side with a finite value of $\Delta=\Delta_{\mathrm{p}}$, although it does not necessarily resemble a BCS-like density of states.

\section{EXPERIMENTAL DATA AND INTERPRETATION}

Figure 1(d) gives an overview of the differential conductance across the point contact for four different devices at zero applied gate voltage and zero magnetic field. At voltages $\left|V_{\mathrm{SN}}\right|>1.5 \mathrm{mV}$, larger than $\Delta_{\mathrm{Nb}}$, the differential conductance is almost constant, and a normal-state resistance of $R_{\mathrm{N}}=160-240 \Omega$ is observed, depending on the measured device. For voltages around $V_{\mathrm{SN}} \simeq 1.1 \mathrm{meV}$ the conductance is slightly enhanced, which is indicated by the black arrows, and then starts to decrease for smaller voltages. Close to 
zero bias, the conductance is enhanced again, resulting in a double-peak structure around $V_{\mathrm{SN}}=0$, with a peak separation of about $100 \mu \mathrm{V}$ for device 1 and slightly different value for the other devices. The red arrows are used to draw attention to a sample-dependent subgap feature. The four devices differ with respect to the shape and length of the HgTe bar underneath the superconductor. Device 1 is symmetric, with width $w=1 \mu \mathrm{m}$ and two open ends. Device 2 has a steplike shape, with partially width $w$ and partially a width of $0.6 \mu \mathrm{m}$. Device 3 is similar, but with the wide "normal" electrode connected to the wide part rather than the narrower part. Finally, device 4 is terminated half way and implies a largely closed $\mathrm{HgTe}$ bar. At present it is not clear whether this should be interpreted as a feature in the relevant nonequilibrium distribution entering Eq. (1) or as reflecting a finite size effect of $\mathrm{HgTe}$ in the spirit of the analysis of Kopnin and Melnikov [19]. Systematic shape-dependent experiments are needed to map and evaluate this dependence accurately and to test the full hypothesis. An asymmetric background for negative and positive biases is observed in all devices. The data can be normalized by multiplying by the normal-state resistance $R_{N}$ measured at $T>T_{c}$, as shown in Fig. 2, to eliminate this slope. We will now discuss the observed behavior in more detail.

\section{A. Low-voltage data: Proximity-induced order parameter}

Close to zero bias, we find a strongly enhanced conductance with a double-peak structure in devices 1-3 and a single peak for the closed bar (device 4). As shown in Fig. 2, this double-peak structure merges at higher temperatures to a bell-shaped curve.

The data in Fig. 2 are taken for device 1, which we will focus on for a detailed analysis. The conductance is normalized with the normal-state resistance $R_{N}$ above the critical temperature $T>T_{c}$. From Fig. 2(a) it is clear that at $4.2 \mathrm{~K}$ an energy gap opens up, which is on the scale of the superconducting niobium gap. Upon lowering the temperature a peak emerges around $V_{\mathrm{SN}}=0$, which splits in two below $500 \mathrm{mK}$.

Figure 2(b) shows the conductance measured at $30 \mathrm{mK}$ for increasing values of magnetic field applied perpendicular to the sample. We verified that the response is independent of the direction of the applied magnetic field. For clarity, a small vertical shift has been removed in the presentation of the data in Fig. 2(b) to highlight that the high-voltage part of the conductance is immune to these magnetic field strengths. Evidently, the central peak can be suppressed completely by applying a magnetic field. We attribute this central bellshaped peak, which evolves into a two-peak structure, to a manifestation of the proximity-induced superconducting order parameter as given by Eq. (2).

For $V_{\mathrm{SN}}=0$ the system is in equilibrium, and the apparent $\Delta$ is the result of electrons in the HgTe bar underneath the niobium film, which are confined in a certain width and length. Their occupation is given by a Fermi-Dirac distribution, and it is part of the superconducting equilibrium reservoir $S_{p}$. For finite voltage bias, the current in $S_{p}$ is carried away as a supercurrent, and importantly, the voltage drop occurring at the interface indicated by $Z \mathrm{p}$ in Fig. 3(a) is due to the difference in electrochemical potentials between $\mathrm{N}$ on the left of $Z_{\mathrm{p}}$ and $\mathrm{S}_{\mathrm{p}}$ on the right of $Z_{\mathrm{p}}$. The scale of the relevant Sharvin resistance
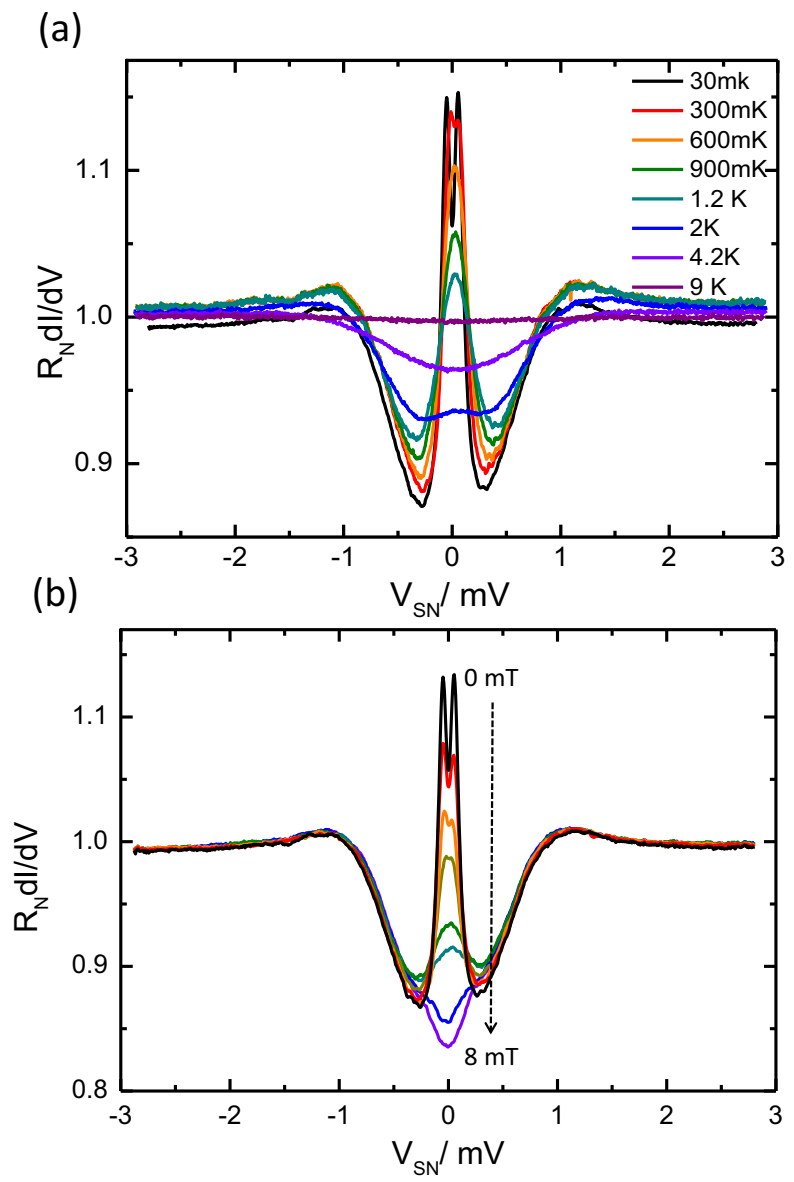

FIG. 2. (a) Conductance of device 1 normalized to the resistance $R_{N}$ at $T=9 \mathrm{~K}$ (purple). At $4.2 \mathrm{~K}$ an energy gap has clearly opened up due to the niobium being superconducting. Upon lowering the temperature a peak emerges around $V_{\mathrm{SN}}=0$, which splits below $500 \mathrm{mK}$. (b) Conductance measured at $30 \mathrm{mK}$ for increasing (small) magnetic field values. This response is independent of the direction of the applied magnetic field. For clarity a small vertical shift has been removed in the presentation of the data to highlight that the high-voltage part of the conductance is immune to these magnetic field strengths.

is controlled by the number of modes at the $Z_{\mathrm{p}}$ location and by the unknown value of $Z_{\mathrm{p}}$. Therefore, the normalization on $R_{N}$ as defined above is not viable in this equilibrium regime.

In Fig. 4(a), data for different temperatures are compared with standard BTK modeling using Eq. (1) (cyan) and the model that explicitly takes the surface states of a 3DTI into account from Ref. [14] (magenta), both leading to very reasonable agreement. In Fig. 4(a) we have renormalized the data differently. We have chosen the conductance value at the edge of the gray zone in Fig. 4(b) as a reasonable approximation of the real value of $R_{N}$ entering Eq. (1). From the comparison shown in Fig. 4(a), we conclude that we find a proximityinduced order parameter $\Delta_{\mathrm{p}}=70 \mu \mathrm{eV}$ for both models.

The fits using Eq. (1) were obtained with a small barrier height $Z_{\mathrm{p}}=0.4$. Here, we have assumed that the proximityinduced order parameter $\Delta_{\mathrm{p}}$ leads to a standard BCS-like behavior of the coefficients $A(E)$ and $B(E)$ as a function of energy and that the normal state is described by a parabolic band 


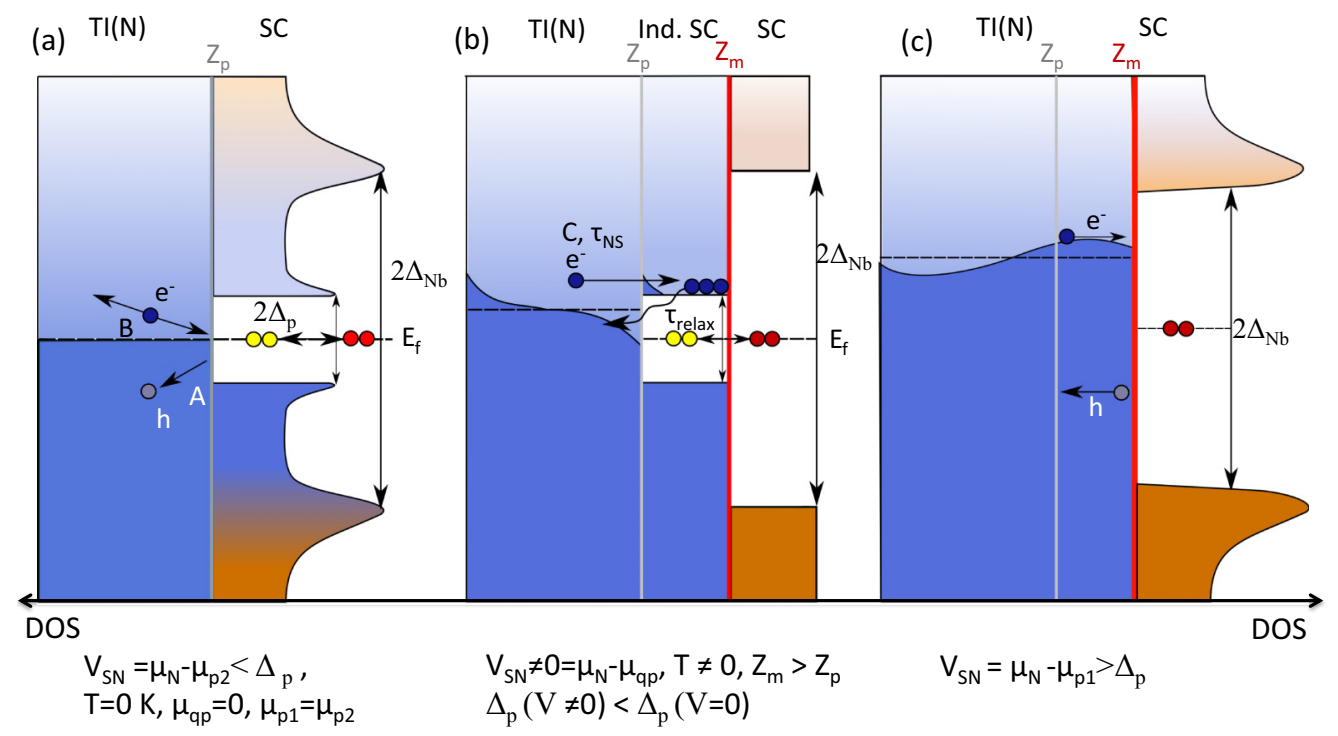

FIG. 3. Semiconductor representation of the NcS system for different bias regimes. The normal part is the topological insulator, the constriction is, initially, characterized by a barrier $Z_{\mathrm{p}}$, and the superconductor is characterized by a pair potential $\Delta_{\mathrm{p}}$. In (a) the system is at zero bias and zero temperature. The voltage difference will emerge at the narrow point contact, and Andreev reflections (normal reflections) are occurring there with probability A (B). The TI-Cooper pairs are phase-coherently coupled to the Nb condensate $\Delta_{\mathrm{Nb}}$. They form one superconducting condensate. In (b) at finite temperature and bias, electrons from higher energies are allowed to enter the proximity-induced superconductor. These "hot" electrons are trapped in the proximitized area because Andreev reflection does not carry entropy. The only relaxation mechanism is by electron-phonon relaxation or by contact with a thermal equilibrium reservoir. So the proximity-induced superconducting state $S_{p}$ is quenched $\left(\Delta_{p} \rightarrow 0\right)$, and the situation as depicted in (c) occurs. Then, at higher voltages transport is measured between a normal reservoir, being the 3DTI HgTe, and the superconductor niobium with an interface resistance characterized by $Z_{\mathrm{m}}$.

dispersion. The model might therefore not capture the microscopic details but is suitable for comparison to other systems.

The treatment of Ref. [14] models the conductance of a NS junction on the surface of a 3DTI, which is exactly appropriate for our experiment. The contact between the normal region and the induced superconducting reservoir is modeled as a square potential barrier, where the dimensionless barrier strength $Z_{\mathrm{p}}$ is defined as the product of the barrier height and width. The subgap tunnel conductance of the NS junction is then an oscillatory function of the barrier strength $Z_{\mathrm{p}}$ and minimum for values $Z_{\mathrm{p}}=(n+1 / 2) \pi$, with $n$ being an integer [20,21]. By applying this model to our experimental data, a rather large barrier can be used. The enhanced conductance can then be seen as a signature of the helical surface states where highly transparent modes are always expected due to Klein tunneling.

We interpret the low-voltage data as a probe of the induced superconducting state in the 3DTI of strained HgTe. There is no reason to expect a priori an $s$-wave order parameter. In fact, we expect deviations, such as those predicted by Burset et al. [14]. Since the actual spectra depend on several parameters, a larger data set is needed to provide a reliable analysis to show the influence of the helical Dirac nature of the surface states. Nevertheless, this open question does not affect the conclusion that we can draw with respect to the identification of the regime, where spectroscopy of the induced superconducting state can reliably be performed.

\section{B. High-voltage data: Niobium order parameter}

For voltages larger than $0.5 \mathrm{meV}$, the conductance curves in Fig. 2(b) all superimpose, if we except the central part interpreted as the proximity-induced order parameter. The data outside the central part can no longer be interpreted as the conductance of a NcS point contact at $Z_{\mathrm{p}}$. The electronic states in the HgTe bar underneath the niobium are no longer correlated as expressed in Eq. (2). For increasing voltage at the location $Z_{\mathrm{p}}$, higher-energy quasiparticles are injected into the HgTe bar as depicted in Fig. 3(b). They cannot escape into an equilibrium reservoir because of the large gap of the superconductor niobium and the fact that Andreev reflections do not exchange heat. Therefore, $f_{0}(E)$ in Eq. (2) becomes a nonequilibrium distribution with relatively hot electrons, which leads in general to the destruction of the proximity-induced order parameter $\Delta_{\mathrm{p}}$ in the same way as a small magnetic field quenches this induced superconducting state. Hence, beyond a voltage of about $0.5 \mathrm{meV}$ the system has changed, and we are left with a nonsuperconducting $\mathrm{HgTe}$ bar in contact with niobium [as shown in Fig. 3(c)] with an interface with an unknown transmissivity parametrized by $Z_{\mathrm{m}}$.

The change in conductance around $1.1 \mathrm{mV}$ is now naturally attributed to the superconducting gap of the niobium film. The conductance increases slightly, as expected at the superconducting gap edge. For smaller voltages the conductance decreases, an indication of dominant normal reflections over Andreev reflections $(B / A>1)$. As shown in Fig. 4(b), we are able to achieve fairly good qualitative agreement with a BTK analysis as well for this outer gap, using a quite large barrier $Z_{\mathrm{m}}=1.1$ and $\Delta_{\mathrm{Nb}}=0.8 \mathrm{meV}$, indicating a relatively low transparency of the $\mathrm{Nb} / \mathrm{HgTe}$ interface. We also need to use a relatively large broadening parameter $\Gamma=0.7 \Delta_{\mathrm{Nb}}$, which could be caused by the large contact area and spatial gradients at the $\mathrm{Nb} / \mathrm{HgTe}$ interface. 
(a)

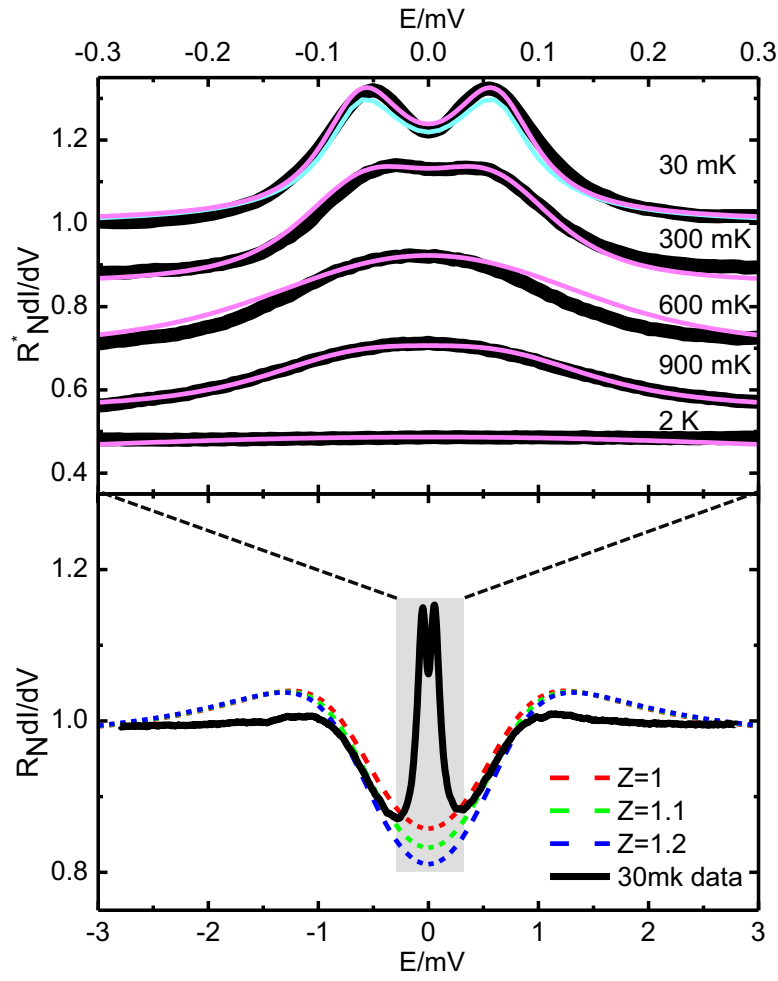

FIG. 4. In (a) the central split peak [gray zone in (b)] is compared to an analysis using Eq. (1) (cyan) with a fixed value of $Z_{p}=0.4$ and a broadening parameter $\Gamma \approx 0.025 \Delta_{\mathrm{p}}$. The magenta lines show a comparison with the model developed in Ref. [14] with a broadening parameter $\Gamma<0.015 \Delta_{\mathrm{p}}$. The value of $\Delta_{\mathrm{p}}$ in both models is $70 \mu \mathrm{eV}$. In (a) we have abandoned the normalization of the data on $R_{N}$ at high voltages and in the normal state. Instead, we have chosen to take the conductance value at the edge of the gray zone. The precise value is a bit arbitrary but should be close to this value. The curves are offset for better visibility. (b) Conductance of device 1 normalized with the normal-state resistance $R_{N}$ above the critical temperature $T>T_{c}$ at $30 \mathrm{mK}$. The gray area indicates the voltage range where we assume an equilibrium proximity-induced superconducting state. The dashed lines show fits using Eq. (1) for three different $Z_{\mathrm{m}}$ parameters and a broadening of $0.7 \Delta_{\mathrm{Nb}}$.

\section{GATE DEPENDENCE OF THE CONDUCTANCE}

The previous data are all obtained on the electron side $(n$ type), in which the mobility is high. In Fig. 5(a) conductance data are shown for different gate voltages from +1 to $-4 \mathrm{~V}$, for which the 3DTI changes from $n$ - to $p$-type conduction. The curves are normalized to the resistance $R_{N}\left(T>T_{c}\right)$ for each gate voltage individually. The behavior of the normal-state resistance of device 2 versus the gate voltage is comparable to the reference Hall bar for which we are able to tune the density from initially $n$ doped over the charge neutrality point into the hole-dominated regime. We distinguish two regimes; from 1 to about $-1 \mathrm{~V}$ the device is in the $n$-conducting regime. In this regime the mobility is high, and the point contact is expected to be ballistic. By tuning into the $p$ regime the mobility decreases by about a factor of 10 , and the mean free path is now smaller than the size of the point contact and therefore is expected to be in the diffusive regime.
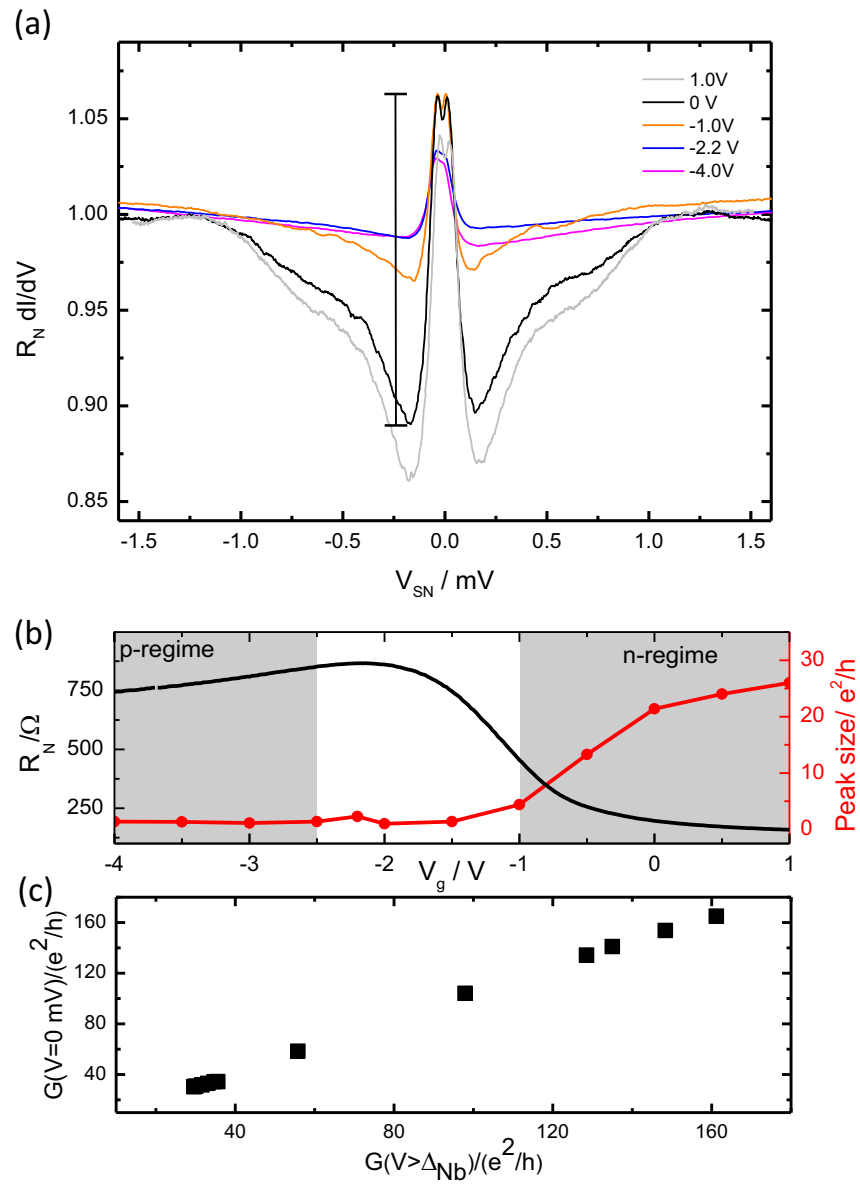

FIG. 5. (a) Gate dependence of normalized conductance of device 2 at $B=0 \mathrm{~T}$ from 1 to $-4 \mathrm{~V}$. The black bar indicates how the height of the central peak is evaluated in (b). (b) Normal-state resistance $R_{N}$ versus gate voltage (black) and size of the peak (red) defined as indicated in (a) by the black bar for $V_{g}=0$. (c) Normal-state conductance versus zero-bias conductance.

From the conductance curves [Fig. 5(a)] it is clear that we no longer observe a signature of the niobium pairing potential in the $p$ regime. Upon changing the gate voltage, features at the scale of the niobium gap disappear upon approaching the Dirac point (at $-2.2 \mathrm{~V}$ ). The only significant voltagedependent feature is around $\pm 100 \mu \mathrm{eV}$. We assume that this observation is a sign that the $\mathrm{NcS}$ point contact is probing the induced superconducting state of the HgTe bar in a diffusive proximity system, leading to a minigap. The height of the zero-bias anomaly as a function of gate voltage is quantified using Fig. 5(a) by defining $d I / d V_{T=30 \mathrm{mK}}-d I / d V_{T>T_{c}}$ and plotted in Fig. 5(b) as red dots. The amplitude is several tens of $e^{2} / h$ in the $n$-conducting regime and decreases continuously up to the maximum in the resistance region, where it saturates at a value of $1-2 e^{2} / h$ depending on the sample.

\section{GENERAL REMARK ABOUT OUR ANALYSIS}

The analysis of our data has led us to discuss the conductance data resulting from the transport through three different electron systems $\left(\mathrm{N}, \mathrm{S}_{\mathrm{p}}\right.$, and $\mathrm{S}_{\mathrm{m}}$ ), separated by two interfaces of transparency, $Z_{\mathrm{p}}$ and $Z_{\mathrm{m}}$. Following Beenakker [22], it is 
assumed that any contact between a normal reservoir and a superconducting reservoir is given by

$$
G_{S}=2 G_{0} \frac{G_{N}^{2}}{\left(2 G_{0}-G_{N}\right)^{2}},
$$

with $G_{0}=2 e^{2} / h$ being the quantum unit of conductance, $G_{N}$ being the conductance in the normal state, and $G_{S}$ being the conductance with one of the electrodes superconducting. This expression is the zero-voltage limit of the classical BTK formula for different values of transmissivity $Z$. In order to calculate $G_{S}$, often, the conductance at $V>\Delta_{s}$ is used as $G_{N}$ [see also Fig. 5(c)], implying that this experimental value is independent of the applied bias. The most important implication in our case is that one measures at high voltages not a proximity-induced superconducting gap, but rather the parent superconductor. We suggest that the low-voltage data should be understood by acknowledging that the scattering region and the equilibrium reservoirs at $V_{\mathrm{SN}}=0$ should be defined differently from those at higher voltages, such as, in our case, $V>0.8 \mathrm{meV}$. This distinction is, in general, not specific to our case but should apply to other topological systems, for example, the one studied in Kjaergaard et al. [23] and Suominen et al. [24], and might explain deviations from expected behavior in these two papers.

\section{CONCLUSIONS}

In conclusion, we have carried out transport spectroscopy of the proximity-induced pair potential of a niobium-covered bar of strained HgTe, which has been demonstrated to be a 3DTI. In analyzing the data we allow for a finite pairing potential in the st $\mathrm{HgTe}$, in contrast to the commonly made quantum transport simplification introduced by Lambert [25] and Beenakker [22], in which the properties are assumed to be controlled exclusively by the scattering in the structure. In addition, we take into account how to identify the relevant distribution function over the energies, implying the relevance of a nonequilibrium distribution function in analyzing the data. These results are an important step towards a better understanding and engineering of topological superconductivity and may serve as a building block for further analysis of the $4 \pi$ Josephson effect as reported in Refs. [8-10].

\section{ACKNOWLEDGMENTS}

We would like to thank A. Akhmerov, W. Belzig, F. S. Bergeret, and B. Trauzettel for many helpful discussions. The work at Würzburg was financially supported by the German Research Foundation DFG via SFB 1170 "ToCoTronics" and SPP 1666, the state of Bavaria (Institute for Topological Insulators and the Elitenetzwerk Bayern), and the European Research Council (advanced grant projects 3-TOP and 4TOPS). T.M.K. acknowledges support from the European Research Council Advanced Grant No. 339306 (METIQUM) and from the Ministry of Education and Science of the Russian Federation, Contract No. 14.B25.31.0007 of 26 June 2013, and T.M.K. and E.B. thank the Alexander von Humboldt Stiftung. P.B. acknowledges support from the European Union's Marie Skłodowska-Curie Grant Agreement No. 743884.
[1] E. Wolf, Principles of Electron Tunneling Spectroscopy, 2nd ed. (Oxford University Press, Oxford, 2012).

[2] G. E. Blonder, M. Tinkham, and T. M. Klapwijk, Phys. Rev. B 25, 4515 (1982).

[3] D. Daghero and R. S. Gonnelli, Supercond. Sci. Technol. 23, 43001 (2010).

[4] E. Wolf and G. Arnold, Phys. Rep. 91, 31 (1982).

[5] E. Scheer, W. Belzig, Y. Naveh, M. H. Devoret, D. Esteve, and C. Urbina, Phys. Rev. Lett. 86, 284 (2001).

[6] M. Kjaergaard, F. Nichele, H. J. Suominen, M. P. Nowak, M. Wimmer, A. R. Akhmerov, J. A. Folk, K. Flensberg, J. Shabani, C. J. Palmstrøm, and C. M. Marcus, Nat. Commun. 7, 12841 (2016).

[7] H. Zhang, Ö. Gül, S. Conesa-Boj, M. P. Nowak, M. Wimmer, K. Zuo, V. Mourik, F. K. de Vries, J. van Veen, M. W. A. de Moor, J. D. S. Bommer, D. J. van Woerkom, D. Car, S. R. Plissard, E. P. A. M. Bakkers, M. Quintero-Pérez, M. C. Cassidy, S. Koelling, S. Goswami, K. Watanabe, T. Taniguchi, and L. P. Kouwenhoven, Nat. Commun. 8, 16025 (2017).

[8] J. Wiedenmann, E. Bocquillon, R. S. Deacon, S. Hartinger, O. Herrmann, T. M. Klapwijk, L. Maier, C. Ames, C. Brüne, C. Gould, A. Oiwa, K. Ishibashi, S. Tarucha, H. Buhmann, and L. W. Molenkamp, Nat. Commun. 7, 10303 (2016).

[9] E. Bocquillon, R. S. Deacon, J. Wiedenmann, P. Leubner, T. M. Klapwijk, C. Brüne, K. Ishibashi, H. Buhmann, and L. W. Molenkamp, Nat. Nanotechnol. 12, 137 (2017).
[10] R. S. Deacon, J. Wiedenmann, E. Bocquillon, F. Domínguez, T. M. Klapwijk, P. Leubner, C. Brüne, E. M. Hankiewicz, S. Tarucha, K. Ishibashi, H. Buhmann, and L. W. Molenkamp, Phys. Rev. X 7, 021011 (2017).

[11] M. Gurvitch, M. A. Washington, and H. A. Huggins, Appl. Phys. Lett. 42, 472 (1983).

[12] M. Snelder, A. A. Golubov, Y. Asano, and A. Brinkman, J. Phys.: Condens. Matter 27, 315701 (2015).

[13] P. Burset, F. Keidel, Y. Tanaka, N. Nagaosa, and B. Trauzettel, Phys. Rev. B 90, 085438 (2014).

[14] P. Burset, B. Lu, G. Tkachov, Y. Tanaka, E. M. Hankiewicz, and B. Trauzettel, Phys. Rev. B 92, 205424 (2015).

[15] C. Brüne, C. X. Liu, E. G. Novik, E. M. Hankiewicz, H. Buhmann, Y. L. Chen, X. L. Qi, Z. X. Shen, S. C. Zhang, and L. W. Molenkamp, Phys. Rev. Lett. 106, 126803 (2011).

[16] C. Brüne, C. Thienel, M. Stuiber, J. Böttcher, H. Buhmann, E. G. Novik, C.-X. Liu, E. M. Hankiewicz, and L. W. Molenkamp, Phys. Rev. X 4, 041045 (2014).

[17] D. Averin and A. Bardas, Phys. Rev. Lett. 75, 1831 (1995).

[18] A. M. Black-Schaffer and A. V. Balatsky, Phys. Rev. B 86, 144506 (2012).

[19] N. B. Kopnin and A. S. Melnikov, Phys. Rev. B 84, 064524 (2011).

[20] S. Bhattacharjee and K. Sengupta, Phys. Rev. Lett. 97, 217001 (2006).

[21] J. Linder and A. Sudbø, Phys. Rev. B 77, 064507 (2008).

[22] C. W. J. Beenakker, Phys. Rev. B 46, 12841 (1992). 
[23] M. Kjaergaard, H. J. Suominen, M. P. Nowak, A. R. Akhmerov, J. Shabani, C. J. Palmstrøm, F. Nichele, and C. M. Marcus, Phys. Rev. Appl. 7, 034029 (2017).
[24] H. J. Suominen, M. Kjaergaard, A. R. Hamilton, J. Shabani, C. J. Palmstrøm, C. M. Marcus, and F. Nichele, arXiv:1703.03699. [25] C. Lambert, J. Phys.: Condens. Matter 3, 6579 (1991). 